\title{
ATG16L1 T300A polymorphism is associated with Crohn's disease in a Northwest Greek cohort, but ECM1 T130M and G290S polymorphisms are not associated with ulcerative colitis
}

\author{
Vasileios E. Tsianos ${ }^{\mathrm{a}}$, Charilaos Kostoulas ${ }^{\mathrm{b}}$, Maria Gazoulic, Stathis Frillingos ${ }^{\mathrm{d}}$, Ioannis Georgiou ${ }^{\mathrm{b}}$, \\ Dimitrios K. Christodoulou' ${ }^{e}$ Konstantinos H. Katsanos ${ }^{e}$, Epameinondas V. Tsianos ${ }^{f}$
}

School of Health Sciences, University of Ioannina; Medical School, National and Kapodistrian University of Athens, Greece

\section{Abstract}

\begin{abstract}
Background Crohn's disease (CD) and ulcerative colitis (UC) are well-described disease entities with unknown etiopathogenesis. Environmental, genetic, gut microbiota, and host immune response correlations have been implicated. The role of susceptibility gene polymorphisms, such as ATG16L1 T300A and ECM1 T130M and G290S, is well-described, although controversial findings have been reported.

Methods Two hundred five patients with inflammatory bowel disease (108 CD and 97 UC), and 223 healthy blood donors (control group) from the Northwest Greece region were genotyped for rs2241880 (T300A), rs3737240 (T130M) and rs13294 (G290S) single nucleotide polymorphisms. Genotyping was performed using the real-time polymerase chain reaction method.

Results The frequency of $\mathrm{G}$ allele was significantly higher in $\mathrm{CD}$ patients compared to the control group $(\mathrm{P}=0.029$; odds ratio $[\mathrm{OR}] 1.45,95 \%$ confidence interval [CI] 1.04-2.03). Carriers of two $\mathrm{G}$ alleles (T300A), compared to those carrying only one, were 1.3 times more susceptible to CD ( $\mathrm{P}=0.022$; OR $2.45,95 \% \mathrm{CI} 1.14-5.27$ ). In CD patients, the presence of the T300A polymorphism indicates a possible protective effect against developing a penetrating (B3) phenotype, while in UC patients, presence of the T300A polymorphism, indicates a possible protective effect against developing joint-involving extraintestinal manifestations.
\end{abstract}

Conclusion Our study found a significant association of the T300A polymorphism with CD susceptibility, suggesting that CD occurrence in our population has a strong genetic background, with the T300A G allele having an additive effect.

Keywords Inflammatory bowel disease, Crohn's disease, ulcerative colitis, ATG16L1, ECM1

Ann Gastroenterol 2019; 32 (6): 1-7

\begin{abstract}
${ }^{a}$ Research Laboratory of Immunology, Faculty of Medicine, School of Health Sciences, University of Ioannina (Vasileios E. Tsianos); ${ }^{b}$ Laboratory of Medical Genetics in Clinical Practice, Faculty of Medicine, School of Health Sciences, University of Ioannina (Charilaos Kostoulas, Ioannis Georgiou); 'Laboratory of Biology, Medical School, National and Kapodistrian University of Athens (Maria Gazouli); ${ }^{\mathrm{d} L a b o r a t o r y}$ of Biological Chemistry, Faculty of Medicine, School of Health Sciences, University of Ioannina (Stathis Frillingos); ${ }^{e}$ Department of Gastroenterology, University Hospital of Ioannina, Faculty of Medicine, School of Health Sciences, University of Ioannina (Dimitrios K. Christodoulou, Konstantinos H. Katsanos); fFaculty of Medicine, School of Health Sciences, University of Ioannina (Epameinondas V. Tsianos), Greece

\section{Conflict of Interest: None}

Correspondence to: Vasileios E. Tsianos, MD, Biologist, MSc, Research Laboratory of Immunology, Faculty of Medicine, School of Health Sciences, University of Ioannina, Dourouti University Campus, 45110 Ioannina, Greece, e-mail: vtsiano@gmail.com

Received 31 July 2019; accepted 14 October 2019; published online 21 November 2019

DOI: https://doi.org/10.20524/aog.2019.0434

\section{Introduction}

Inflammatory bowel disease (IBD) comprises 2 idiopathic relapsing and remitting disorders of the gastrointestinal tract: Crohn's disease (CD) and ulcerative colitis (UC). They are multifactorial, with environmental, genetic, gut microbiota, and host immune response correlations contributing to their etiology [1]. Genetic predisposition to the disease is provided by multiple genes and their distinct allele variants, known as single nucleotide polymorphisms (SNPs). To date, more than 200 susceptible loci have been identified, on a genome-wide scale, representing various pathways in IBD development [2,3]. Furthermore, the role of proteins involved in inflammation, gut defense and epithelial barrier permeability participating in IBD pathogenesis has been elucidated by numerous studies [4,5], and several studies have noted the effect of the genetic background on the phenotype of the disease. For example, in CD patients, carriage of any of the NOD2 and JAK2 variants has been found to predict a 
more complicated disease course, with ileal involvement and stenosing behavior, while possession of any of NOD2 variants is protective against colonic disease [6]. Additionally, ileal CD has also been associated with ATG16L1 variants [7]. On the other hand, in UC patients, severe disease behavior has been associated with polymorphisms of major histocompatibility complex genes [8], and azathioprine use with ECM1 gene polymorphisms [9].

It has been long known that autophagy-related 16-like 1 gene (ATG16L1), and especially its T300A (rs2241880) allele, is associated with susceptibility to CD but not to UC [10-12]. Nevertheless, over the years, various studies on T300A have shown a divergence from this universal susceptibility pattern and variations among different population groups have been widely reported [13-17]. Apart from the correlation between the occurrence of CD and the presence of ATG16L1 polymorphisms, strong evidence of a predisposition to certain phenotypes of T300A carriers also exists, such as ileal CD in T300A carriers [7].

Likewise, extracellular matrix protein-1 (ECM1), a protein involved in epithelial barrier formation, has been associated with UC but not CD $[9,18]$. Inflammation in UC is limited only to the mucosal surface, and polymorphisms of genes that regulate epithelial barrier permeability play an important role in UC pathogenesis [19]. In particular, polymorphisms of the ECM1 gene lead to tissue injury, resulting in intestinal ulcers and scarring in UC patients [20]. Two ECM1 SNPs, rs3737240 (T130M) and rs13294 (G290S), are strongly associated with UC; however, a study from China showed no connection with the disease [21].

Our study area, Northwest (NW) Greece, has remarkable characteristics compared to other Greek regions, as has been previously reported [22]. It used to be a secluded area where population migration was limited, and that might have been crucial in shaping the genetic pool composition. Exceptional in this area is the continuous low incidence of CD compared to UC, despite the phenomenal rise of CD incidence in recent years [23]. Moreover, a relevant study in the past, examining NOD2/CARD15 variants in the aforementioned area [24], showed no coherence with 2 other Greek studies [25,26].

Given the role of $A T G 16 L 1$ and ECM1 polymorphisms in differentiating CD from UC, as well as the discrete associations between SNPs and disease phenotype, and taking into consideration the particularity of our study area, we aimed to evaluate whether our findings correlate with existing data from other regions worldwide and if there is a predictive and/or prognostic association with disease development or a specific clinical phenotype.

\section{Patients and methods}

\section{Study population}

Our study population $(\mathrm{n}=428)$ consisted of 205 unrelated IBD patients (108 CD and 97 UC patients) who attended the Outpatient Clinic and the Gastroenterology Department of the University Hospital of Ioannina, Greece, and 223 unrelated healthy blood donors (control group) from the University Hospital of Ioannina's Blood Bank. All study subjects were from the NW Greece region and are of Caucasian ethnicity. Demographic and clinical data of the study subjects are presented in Table 1. Diagnosis of either CD or UC was based on standard clinical, endoscopic, radiological and histological criteria $[27,28]$. Both CD phenotype (age at onset, disease location, and behavior) and UC phenotype (extent and severity) were determined according to the Montreal Classification [29]. The presence of extraintestinal manifestations (EIMs) and/or other autoimmune disease was established by relevant specialists. Recruitment of the control group subjects was based on their not having any gut- or liver-related disease. All subjects were informed about the nature of the study and signed the informed consent form.

\section{Genotyping}

Genomic DNA was extracted from whole blood samples using a Nucleospin Blood kit (Macherey-Nagel, Germany), according to the manufacturer's protocol. Three SNPs of 2 genes were investigated in this study: namely, rs2241880 (T300A) of ATG16L1 and rs3737240 (T130M) and rs13294 (G290S) of ECM1. Genotyping was carried out using the RotorGene 3000 RealTime-PCR system (Corbett Research, Australia).

The following oligonucleotide primers and SNP-specific probes were used: ATG16L1-T300A-Fw: 5'-TGA AGC ATA CTT ACG AAG ACA CAC-3', ATG16L1-T300A-Rv: 5'-TGT CTC TTC CTT CCC AGT CC-3', ATG16L1-T300A-T: 5'-CCA GAA CCA GGA TGA GTA TCC ACA T-3', ATG16L1T300A-C: 5'-CAG AAC CAG GAT GAG CAT CCA CAT-3', ECM1-T130M-Fw: 5'-CCC CAG ATT CTT TCA ATC CTC-3', ECM1-T130M-Rv: 5'-AGG ACT CAG GTT CTG GAT GG3', ECM1-T130M-C: 5'-TTT CCC CAT TCC AGG AAC GCC AGC TCC ATT-3', ECM1-T130M-T: 5'-TTT CCC CAT TCC AGG AAT GCC AGC TCC ATT-3', ECM1-G290S-Fw: 5'-CCC AAC TAT GAC CGG GAC-3', ECM1-G290S-Rv: 5'-GCA ACT TAC TGC TTG GTG AG-3', ECM1-G290S-G: 5'-CTT GAC CAT TGA CAT CGG TCG AG-3', ECM1-G290S-A: 5'-CTT GAC CAT TGA CAT CAG TCG AGT C-3'.

The total reaction volume used for the RT-PCR assay was $20 \mu \mathrm{L}$, consisting of $1 \mu \mathrm{L}$ genomic DNA (65 ng), $10 \mu \mathrm{L}$ Mastermix (Kapa Probe Fast qPCR kit), $7.8 \mu \mathrm{L}$ PCR-grade water, $0.4 \mu \mathrm{L}$ forward primer, $0.4 \mu \mathrm{L}$ reverse primer and $0.2 \mu \mathrm{L}$ of each of the $2 \mathrm{SNP}$-specific probes. The cycling protocol was as follows: enzyme activation at $95^{\circ} \mathrm{C}$ for $3 \mathrm{~min}$, followed by 40 2-step cycles of denaturation at $95^{\circ} \mathrm{C}$ for $3 \mathrm{sec}$ and annealingelongation at $60^{\circ} \mathrm{C}$ for $20 \mathrm{sec}$. The protocol was applied to all samples for all 3 SNPs. Following an initial run, 3 samples from each SNP (one wildtype, one mutant and one heterozygote) were verified by dsDNA sequencing and subsequently used as control samples in each and every run.

Oligonucleotide synthesis and dsDNA sequencing services were performed by VBC-Biotech Services GmbH (Vienna, Austria). Allelic discrimination was based on the RotorGene 3000 software. 
Table 1 Demographic and clinical characteristics of IBD patients

\begin{tabular}{|c|c|c|}
\hline Characteristics & $\mathrm{CD}(\mathrm{N}=108)$ & $\mathrm{UC}(\mathrm{N}=97)$ \\
\hline Sex (male/female) & $66 / 42$ & $56 / 41$ \\
\hline $\begin{array}{l}\text { Age at diagnosis (mean, } \pm \text { SD, range) } \\
\leq 16 \\
17-40 \\
>40\end{array}$ & $\begin{array}{c}32.7 \pm 13.4,(13-64) \\
6(5.6 \%) \\
76(70.4 \%) \\
26(24.1 \%)\end{array}$ & $\begin{array}{c}39.3 \pm 14.7,(15-83) \\
2(2.1 \%) \\
50(51.5 \%) \\
45(46.4 \%)\end{array}$ \\
\hline Smoking & $50(46.3 \%)$ & $41(42.3 \%)$ \\
\hline $\begin{array}{l}\text { Disease location } \\
\text { L1 - Ileal } \\
\text { L2 - Colonic } \\
\text { L3 - Ileocolitis } \\
\text { L4 - Upper gastrointestinal }\end{array}$ & $\begin{array}{c}33(30.6 \%) \\
32(29.6 \%) \\
43(39.8 \%) \\
6(5.6 \%)\end{array}$ & \\
\hline $\begin{array}{l}\text { Disease behavior } \\
\text { B1 - Nonstricturing, nonpenetrating } \\
\text { B2 - Structuring } \\
\text { B3 - Penetrating } \\
\text { B2+B3 } \\
\text { p - Perianal disease }\end{array}$ & $\begin{array}{l}54(50.0 \%) \\
22(20.4 \%) \\
21(19.4 \%) \\
11(10.2 \%) \\
26(24.1 \%)\end{array}$ & \\
\hline $\begin{array}{l}\text { UC extent } \\
\text { E1 - ulcerative proctitis } \\
\text { E2 - Left sided } \\
\text { E3 - pancolitis }\end{array}$ & & $\begin{array}{l}10(10.3 \%) \\
61(62.9 \%) \\
26(26.8 \%)\end{array}$ \\
\hline $\begin{array}{l}\text { UC severity } \\
\text { Mild/Moderate } \\
\text { Severe }\end{array}$ & & $\begin{array}{l}73(75.3 \%) \\
24(24.7 \%)\end{array}$ \\
\hline $\begin{array}{l}\text { EIMs } \\
1 \text { EIM } \\
>1 \text { EIMs } \\
\text { Joint } \\
\text { Osteoporosis } \\
\text { Skin/Oral } \\
\text { Ocular }\end{array}$ & $\begin{array}{c}67(62 \%) \\
37(34.3 \%) \\
30(27.8 \%) \\
45(41.7 \%) \\
18(16.7 \%) \\
26(24.1 \%) \\
10(9.3 \%)\end{array}$ & $\begin{array}{c}46(47.4 \%) \\
21(21.6 \%) \\
25(25.8 \%) \\
32(33.0 \%) \\
7(7.2 \%) \\
18(18.6 \%) \\
10(10.3 \%)\end{array}$ \\
\hline Other autoimmune disease & $6(5.6 \%)$ & $1(1.0 \%)$ \\
\hline Operated & $16(14.8 \%)$ & $3(3.1 \%)$ \\
\hline Anti-TNF- $\alpha$ & $63(65.6 \%)$ & $33(34.4 \%)$ \\
\hline Cholecystectomy (post-diagnosis) & $7(6.5 \%)$ & $6(6.2 \%)$ \\
\hline Appendectomy (pre-diagnosis) & $20(18.5 \%)$ & $11(11.3 \%)$ \\
\hline Tonsillectomy (pre-diagnosis) & $16(14.8 \%)$ & $16(16.5 \%)$ \\
\hline
\end{tabular}

IBD, inflammatory bowel disease; $C D$, Crohn's disease; UC, ulcerative colitis; EIMs, extraintestinal manifestations; $N$, number; SD, standard deviation; TNF, tumor necrosis factor

\section{Statistical analysis}

The control group was investigated for conformity with Hardy-Weinberg equilibrium $(\mathrm{P}>0.05)$ in all 3 SNPs. Allele and genotype frequencies among groups were calculated using Fisher's exact test. The additive effect of alleles was tested using binary logistic regression analyses. Association assessment of clinical, demographic and genotypic data was implemented using liner regression analysis or Fisher's exact test, where appropriate. The results were expressed as odds ratios (OR) with a confidence interval of $95 \%(95 \% \mathrm{CI})$. A 2 -sided P-value of $<0.05$ was considered as statistically significant. Statistical analyses were performed using the jamovi software: the jamovi project (2019); jamovi (Version 1.0) (https://www.jamovi. org). Additionally, linkage disequilibrium between rs3737240 and rs13294 of ECM1 was tested using the SNPstats software (http://bioinfo.iconcologia.net).

\section{Results}

One hundred eight CD patients, 97 UC patients and 223 healthy individuals (control group) were genotyped in order to examine possible associations of the 3 SNPs with IBD patients in NW Greece. The healthy control group was in Hardy-Weinberg equilibrium with all 3 SNPs. Allele and 
Table 2 ATG16L1 rs2241880 allele and genotype frequencies in CD, UC and control group (Fisher's exact test, odds ratio and confidence intervals were estimated using allele frequencies in $2 \times 2$ contingency tables)

\begin{tabular}{|c|c|c|c|c|c|c|c|c|c|}
\hline \multicolumn{5}{|c|}{ Alleles } & \multicolumn{5}{|c|}{ Genotypes } \\
\hline $\begin{array}{l}\text { ATG16L1 } \\
\text { (rs2241880) }\end{array}$ & $\mathrm{A}$ & G & $\begin{array}{l}\mathrm{G} \text { allele } \\
\text { freq. (\%) }\end{array}$ & $\mathrm{P}[\mathrm{OR}(95 \% \mathrm{CI})]$ & AA & AG & GG & $\begin{array}{l}\text { GG } \\
\text { genotype } \\
\text { freq. (\%) }\end{array}$ & $\mathrm{P}[\mathrm{OR}(95 \% \mathrm{CI})]$ \\
\hline $\mathrm{CD}$ & 77 & 139 & 64.4 & $0.029[1.45(1.04-2.03)]$ & 11 & 55 & 42 & 38.9 & $0.134[1.48(0.91-2.40)]$ \\
\hline UC & 80 & 114 & 58.8 & $0.436[1.15(0.82-1.61)]$ & 14 & 52 & 31 & 32.0 & $0.733[1.09(0.65-1.83)]$ \\
\hline IBD & 157 & 253 & 61.7 & $0.061[1.30(0.99-1.71)]$ & 25 & 107 & 73 & 35.6 & $0.257[1.29(0.86-1.93)]$ \\
\hline Control group & 199 & 247 & 55.4 & & 43 & 113 & 67 & 30.0 & \\
\hline
\end{tabular}

CD, Crohn's disease; UC, ulcerative colitis; IBD, inflammatory bowel disease; OR, odds ratio; CI, confidence interval

genotype frequencies for the T300A ATG16L1 polymorphism (rs2241880) are presented in Table 2, where AA represents wildtype, AG heterozygotes and GG mutants. The frequency of the $\mathrm{G}$ allele of the T300A polymorphism was $64.4 \%, 58.8 \%$ and $55.4 \%$ in $\mathrm{CD}$, UC and healthy individuals, respectively. When compared to the control group, the frequency of the $G$ allele in Crohn's disease patients was significantly higher $(\mathrm{P}=0.029$; OR 1.45, 95\%CI 1.04-2.03), while it showed no significant association with UC patients.

Investigating the additive effect of the $G$ allele in $C D$ patients, we found that carriers of $2 \mathrm{G}$ alleles (mutant group), compared to those carrying only one $\mathrm{G}$ allele (heterozygotes), were 1.3 times more susceptible to $\mathrm{CD}$ (Table 3 ); the difference was statistically significant (GG: $\mathrm{P}=0.022$; OR $2.45,95 \% \mathrm{CI}$ 1.14-5.27. AG: $\mathrm{P}=0.087$; OR 1.90, 95\%CI 0.91-3.97).

Correspondingly, T130M and G290S ECM1 polymorphisms (rs3737240, rs13294) are shown in Table 4. For T130M, CC represents wildtype, CT heterozygotes and TT mutants, while for G290S, GG represents wildtype, GA heterozygotes and AA mutants. The frequency of the $\mathrm{T}$ allele of $\mathrm{T} 130 \mathrm{M}$ was $46.3 \%$, $39.7 \%$ and $44.2 \%$ for CD, UC and control group, respectively, while the frequency of A allele of G290S mutation was $46.3 \%$, $38.2 \%$ and $43.3 \%$ respectively. No strong associations between either of the 2 SNPs of the ECM1 gene and our study group were found. No additive effect for either of the 2 ECM1 SNPs was found. Furthermore, comparison of the 2 IBD groups (CD and UC) with each other, from a genotypic point of view, showed no significant difference for any of the 3 SNPs ( $\mathrm{P}=0.290$; OR 1.27, 95\%CI 0.85-1.89 for T300A. $\mathrm{P}=0.195$; OR 1.31, 95\%CI 0.88-1.94 for T130M. $\mathrm{P}=0.109$; OR $1.40,95 \% \mathrm{CI}$ 0.94-2.07 for G290S).

Moreover, some interesting genotype-phenotype associations among IBD patients carrying T300A SNP were observed. Specifically, in CD patients, the presence of one or two $\mathrm{G}$ alleles of the T300A polymorphism (AG+GG genotypes) indicated a possible protective effect against developing a penetrating phenotype (B3 behavior according to Montreal classification [29]) ( $\mathrm{P}=0.015$; OR $0.20,95 \% \mathrm{CI} \quad 0.05-0.74)$, while in UC patients, the presence of one or two G alleles of the T300A polymorphism ( $\mathrm{AG}+\mathrm{GG}$ genotypes) indicated a possible protective effect against developing joint-involving EIMs ( $\mathrm{P}=0.038$; OR 0.31, 95\%CI 0.10-0.97; Table 5). However, measure analyses of these associations, performed using the
Table 3 Additive effect of G allele, ATG16L1; T300A (Binary logistic regression analyses)

\begin{tabular}{lccc}
\hline Crohn's disease & P-value & Odds ratio & $95 \% \mathrm{CI}$ \\
\hline AG & 0.087 & 1.90 & $0.91-3.97$ \\
GG & 0.022 & 2.45 & $1.14-5.27$ \\
\hline
\end{tabular}

CI, confidence interval

phi-coefficient $(\Phi)$ test, showed that these findings were of mild association ( $\Phi=0.251$ and $\Phi=0.211$ respectively). Furthermore, in CD patients carrying the T300A SNP (AG+GG genotype) we found an indication of a possible protective effect against the need for cholecystectomy $(\mathrm{P}=0.022$; OR $0.12,95 \% \mathrm{CI}$ $0.02-0.60)$, though with only a mild-to-moderate association $(\Phi=0.284)$. However, the number of $C D$ patients who underwent cholecystectomy (post-diagnosis) was small $(n=7)$.

No association was found between the age at onset, CD location, UC extent and severity, presence of EIMs or other immune disease, need of operation, anti-tumor necrosis factor (TNF)- $\alpha$ treatment, appendectomy or tonsillectomy and any of the three SNPs for either CD or UC patients (data not shown). Also, the 2 polymorphisms of ECM1 gene were not found to be in linkage disequilibrium.

\section{Discussion}

IBD affects millions worldwide and its etiopathology still remains unknown. There is an international scientific consensus that a fine interaction among genetic susceptibility, environmental factors, immune response and gut microbiota may hold the answer. Nevertheless, studies have shown that a global pattern of genetic influence, heritability, environmental triggers and gut fauna does not exist. However, to investigate the matter further, any available data can contribute greatly towards elucidating the true face of IBD; thus, studies from various areas and ethnicities are needed.

This is the first study of ATG16L1 and ECM1 gene polymorphisms in our NW Greece cohort, a previously welldescribed sheltered area [22]. A previous genotypic study from this area focusing on the NOD2/CARD15 gene showed no association between the studied polymorphisms and CD 
Table 4 ECM1 rs3737240 and rs13294 allele and genotype frequencies in CD, UC and control group (Fisher's exact test, odds ratio and confidence intervals were estimated using allele frequencies in $2 \times 2$ contingency tables)

\begin{tabular}{|c|c|c|c|c|c|c|c|c|c|}
\hline \multicolumn{5}{|c|}{ Alleles } & \multicolumn{5}{|c|}{ Genotypes } \\
\hline $\begin{array}{l}E C M 1 \\
\text { (rs3737240) }\end{array}$ & $\mathrm{C}$ & $\mathrm{T}$ & $\begin{array}{l}\mathrm{T} \text { allele } \\
\text { freq. (\%) }\end{array}$ & $\mathrm{P}$ [OR $(95 \% \mathrm{CI})]$ & $\mathrm{CC}$ & $\mathrm{CT}$ & $\mathrm{TT}$ & $\begin{array}{c}\text { TT } \\
\text { genotype } \\
\text { freq. (\%) }\end{array}$ & $\mathrm{P}$ [OR $(95 \% \mathrm{CI})]$ \\
\hline $\mathrm{CD}$ & 116 & 100 & 46.3 & $0.617[1.09(0.79-1.51)]$ & 26 & 64 & 18 & 16.7 & $0.550[0.79(0.43-1.45)]$ \\
\hline UC & 117 & 77 & 39.7 & $0.298[0.83(0.59-1.17)]$ & 34 & 49 & 14 & 14.4 & $0.273[0.67(0.35-1.28)]$ \\
\hline IBD & 233 & 177 & 43.2 & $0.78[0.96(0.73-1.26)]$ & 60 & 113 & 32 & 15.6 & $0.257[0.73(0.44-1.21)]$ \\
\hline $\begin{array}{l}\text { Control } \\
\text { group }\end{array}$ & 249 & 197 & 44.2 & & 71 & 107 & 45 & 20.2 & \\
\hline $\begin{array}{l}\text { ECM1 } \\
\text { (rs13294) }\end{array}$ & G & A & $\begin{array}{l}\text { A allele } \\
\text { freq. (\%) }\end{array}$ & $\mathrm{P}$ [OR $(95 \% \mathrm{CI})]$ & GG & GA & $\mathrm{AA}$ & $\begin{array}{l}\text { AA } \\
\text { genotype } \\
\text { freq. (\%) }\end{array}$ & P [OR (95\% CI)] \\
\hline $\mathrm{CD}$ & 116 & 100 & 46.3 & $0.505[1.13(0.81-1.57)]$ & 26 & 64 & 18 & 16.7 & $0.550[0.79(0.43-1.45)]$ \\
\hline UC & 120 & 74 & 38.2 & $0.257[0.81(0.57-1.14)]$ & 36 & 48 & 13 & 13.4 & $0.159[0.61(0.31-1.20)]$ \\
\hline IBD & 236 & 174 & 42.4 & $0.836[0.97(0.74-1.27)]$ & 62 & 112 & 31 & 15.1 & $0.205[0.71(0.43-1.17)]$ \\
\hline $\begin{array}{l}\text { Control } \\
\text { group }\end{array}$ & 253 & 193 & 43.3 & & 75 & 103 & 45 & 20.2 & \\
\hline
\end{tabular}

$\overline{C D}$, Crohn's disease; UC, ulcerative colitis; IBD, inflammatory bowel disease; OR, odds ratio; CI, confidence interval

Table 5 ATG16L1 T300A Genotype and phenotype associations (Fisher's exact test and correlation test)

\begin{tabular}{lcccccc}
\hline \multirow{2}{*}{$\begin{array}{l}\text { ATG16L1 T300A } \\
\text { (rs2241880) }\end{array}$} & \multicolumn{3}{c}{ Crohn's disease } & & \multicolumn{2}{c}{ Ulcerative colitis } \\
\cline { 2 - 4 } \cline { 5 - 6 } & P-value & OR $(95 \% \mathrm{CI})$ & $\Phi$ & & P-value & OR (95\%CI) \\
\hline $\begin{array}{l}\text { B3 - Penetrating } \\
\text { behavior }\end{array}$ & 0.015 & $0.20(0.05-0.74)$ & 0.251 & & - \\
$\begin{array}{l}\text { Cholecystectomy } \\
\begin{array}{l}\text { Joint-involving } \\
\text { EIMs }\end{array}\end{array}$ & 0.022 & $0.12(0.02-0.60)$ & 0.284 & & - & - \\
\hline
\end{tabular}

P-value: AG+GG vs. AA

OR, odds ratio; CI, confidence interval; $\Phi$, Phi-coefficient; EIMs, extraintestinal manifestations

susceptibility [24], a divergent result compared to most studies from other areas [30,31]. Furthermore, the role of T300A ATG16L1 and T130M and G290S ECM1 gene polymorphisms in the development of $\mathrm{CD}$ and $\mathrm{UC}$, respectively, is wellestablished in the literature $[10,20]$, despite some ethnic variabilities $[13,21]$. Our study replicated the finding that the T300A ATG16L1 polymorphism predisposes to CD in our cohort, also identifying an additive effect of the G allele in CD patients, but failed to demonstrate any association between ECM1 polymorphisms and UC susceptibility.

On the other hand, despite the strong occurrence of the $G$ allele in the CD group compared to the control group, a clear distinction among IBD patients (CD vs. UC analyses) could not be established. Similarly, as mentioned earlier, ECM1 SNP investigation in our population failed to replicate existing data $[9,20]$ and, again, no distinction among IBD patients was found. Hence, in our study group, it is not possible to differentiate the underlying disease (CD or $\mathrm{UC}$ ) based on genotypic-phenotypic associations, probably because of ethnic variations, but more patients and more widely associated susceptibility genes are needed to drive to a definite conclusion.

In contrast, we found some interesting protective associations of certain phenotypes and IBD patients. In the CD group, patients who were carriers (AG and GG patients) of $G$ allele of the T300A polymorphism were found to be associated with a possible protective effect against penetrating behavior (B3 phenotype according to the Montreal classification [29]). This finding disagreed with the results of another study, where the majority of the patients were found to exhibit penetrating behavior [7], suggesting that a potential environmental or ethnicity trigger may be present in our cohort and played a role in developing such a phenotype. Again, in the CD group, carriers of the $\mathrm{G}$ allele (AG and GG patients) of the T300A polymorphism were found to have a mild association with a protective effect against the need for a cholecystectomy. In the literature, gallbladder disease is well described in IBD patients and is mainly associated with Crohn's disease [32], 
though a recent meta-analysis by Zhang et al [33] concluded that, despite the apparent association of $\mathrm{CD}$ and gallbladder disease, other factors, such as $\mathrm{CD}$ location, number of relapses and ileal surgery, were independent variables for developing cholelithiasis; more studies are required to provide a definite answer. Moreover, the $\mathrm{G}$ allele (T300A polymorphism) was found to have a protective effect against joint-involving EIMs in UC patients. As described before by Christodoulou et al [34], and confirmed by this study, EIMs are not rare in our IBD cohort ( $62 \%$ of CD patients and $47.4 \%$ of UC patients) and data from other studies suggest that there is a close genetic correlation between IBD and EIMs [35]. However, such associations between IBD susceptibility genes and EIM occurrence could not be demonstrated in the present study.

When other clinical data (age at onset, CD location, CD behavior, UC extent, UC severity, need of therapeutic operation, anti-TNF- $\alpha$ therapy) were analyzed for any possible linkage with the aforementioned mutations, no significant associations were found, although such associations have been reported in the literature $[7,16,17,36,37]$.

In conclusion, as shown by Tsianos et al in 2003 [23], CD is less frequent than UC in our cohort. Thus, the findings of our current study, concerning the significant association of T300A polymorphism with CD susceptibility, point to a strong genetic background that plays a crucial role in $\mathrm{CD}$ occurrence in our population, and an additive effect of the T300A G allele, though further investigation including more patients and more susceptibility genes will provide a better understanding.

\section{Acknowledgments}

The authors would like to thank the patients who participated in this study. The authors acknowledge the contribution of Kleopatra Garallea and Gioula Georgitsi, for their technical support, and Dr E. Zervou for helping with the sample collection from healthy blood donors.

\section{References}

1. Loddo I, Romano C. Inflammatory bowel disease: genetics, epigenetics, and pathogenesis. Front Immunol 2015;6:551.

2. de Lange KM, Moutsianas L, Lee JC, et al. Genome-wide association study implicates immune activation of multiple integrin genes in inflammatory bowel disease. Nat Genet 2017;49:256-261.

3. Luo Y, de Lange KM, Jostins L, et al. Exploring the genetic architecture of inflammatory bowel disease by whole-genome sequencing identifies association at ADCY7. Nat Genet 2017;49:186-192.

4. Shih DQ, Targan SR. Insights into IBD pathogenesis. Curr Gastroenterol Rep 2009;11:473-480.

5. Van Limbergen J, Radford-Smith G, Satsangi J. Advances in IBD genetics. Nat Rev Gastroenterol Hepatol 2014;11:372-385.

6. Cleynen I, González JR, Figueroa C, et al. Genetic factors conferring an increased susceptibility to develop Crohn's disease also influence disease phenotype: results from the IBDchip European Project. Gut 2013;62:1556-1565.

\section{Summary Box}

\section{What is already known:}

- More than 200 inflammatory bowel disease susceptibility loci have been identified

- The ATG16L1 T300A single nucleotide polymorphism (SNP) is associated with susceptibility to Crohn's disease (CD) but not to ulcerative colitis (UC)

- ECM1 T130M and G290S SNPs are strongly associated with UC but not with CD

\section{What the new findings are:}

- The T300A ATG16L1 polymorphism predisposes to $\mathrm{CD}$ in our cohort

- The $\mathrm{G}$ allele has an additive effect in CD patients

- The T300A SNP has a protective effect against penetrating behavior and cholecystectomy in $\mathrm{CD}$ patients), and against joint-involving extraintestinal manifestations in UC patients

- No association of ECM1 polymorphisms with UC susceptibility was found in our cohort

7. Prescott NJ, Fisher SA, Franke A, et al. A nonsynonymous SNP in ATG16L1 predisposes to ileal Crohn's disease and is independent of CARD15 and IBD5. Gastroenterology 2007;132:1665-1671.

8. de la Concha EG, Fernandez-Arquero M, Lopez-Nava G, et al. Susceptibility to severe ulcerative colitis is associated with polymorphism in the central MHC gene IKBL. Gastroenterology 2000;119:1491-1495.

9. Adalı G, Ersoy Tunalı N, Yorulmaz E, et al. Extracellular matrix protein 1 gene rs3737240 single nucleotide polymorphism is associated with ulcerative colitis in Turkish patients. Turk $J$ Gastroenterol 2017;28:254-259.

10. Hampe J, Franke A, Rosenstiel P, et al. A genome-wide association scan of nonsynonymous SNPs identifies a susceptibility variant for Crohn disease in ATG16L1. Nat Genet 2007;39:207-211.

11. Lee JC, Biasci D, Roberts R, et al; UK IBD Genetics Consortium. Genome-wide association study identifies distinct genetic contributions to prognosis and susceptibility in Crohn's disease. Nat Genet 2017;49:262-268.

12. Rioux JD, Xavier RJ, Taylor KD, et al. Genome-wide association study identifies new susceptibility loci for Crohn disease and implicates autophagy in disease pathogenesis. Nat Genet 2007;39:596-604.

13. Walker DG, Bancil AS, Rai PS, et al. Ethnic variation in the frequency of IBD related polymorphisms in IRGM, ATG16L1 and IL23R. Gut 2011;60:A223.

14. Yang SK, Park M, Lim J, et al. Contribution of IL23R but not ATG16L1 to Crohn's disease susceptibility in Koreans. Inflamm Bowel Dis 2009;15:1385-1390.

15. Pugazhendhi S, Baskaran K, Santhanam S, Ramakrishna BS. Association of ATG16L1 gene haplotype with inflammatory bowel disease in Indians. PLoS One 2017;12:e0178291.

16. Lakatos PL, Szamosi T, Szilvasi A, et al. ATG16L1 and IL23 receptor (IL23R) genes are associated with disease susceptibility in 
Hungarian CD patients. Dig Liv Dis 2008;40:867-873.

17. Aida I, Meddour Y, Kadiri H, et al. T300A variant of AT16L1 gene in a cohort of Algerian Crohn disease patients. Curr Res Transl Med 2018;66:9-14.

18. Thompson AI, Lees CW. Genetics of ulcerative colitis. Inflamm Bowel Dis 2011;17:831-848.

19. McCole DF. IBD candidate genes and intestinal barrier regulation. Inflamm Bowel Dis 2014;20:1829-1849.

20. Fisher SA, Tremelling M, Anderson CA, et al; Wellcome Trust Case Control Consortium. Genetic determinants of ulcerative colitis include the ECM1 locus and five loci implicated in Crohn's disease. Nat Genet 2008;40:710-712.

21. Shi J, Zhou L, Zhernakova A, et al. Haplotype-based analysis of ulcerative colitis risk loci identifies both IL2 and IL21 as susceptibility genes in Han Chinese. Inflamm Bowel Dis 2011;17:2472-2479.

22. Tsianos EV, Masalas CN, Merkouropoulos M, Dalekos GN, Logan RF. Incidence of inflammatory bowel disease in north west Greece: rarity of Crohn's disease in an area where ulcerative colitis is common. Gut 1994;35:369-372.

23. Tsianos EV, Katsanos KH, Christodoulou D, et al. Continuing low incidence of Crohn's disease in Northwest Greece. Dig Liv Dis 2003;35:99-103.

24. Economou M, Filis G, Tsianou Z, et al. Crohn's disease incidence evolution in North-western Greece is not associated with alteration of NOD2/CARD15 variants. World J Gastroenterol 2007;13:5116-5120.

25. Roussomoustakaki M, Koutroubakis I, Vardas EM, et al. NOD2 insertion mutation in a Cretan Crohn's disease population. Gastroenterology 2003;124:272-273.

26. Gazouli M, Zacharatos P, Mantzaris GJ, et al. Association of NOD2/ CARD15 variants with Crohn's disease in a Greek population. Eur J Gastroenterol Hepatol 2004;16:1177-1182.

27. Podolsky DK. Inflammatory bowel disease. $N$ Engl J Med
2002;347:417-429.

28. Lennard-Jones JE. Classification of inflammatory bowel disease. Scand J Gastroenterol 1989;24(Suppl 170):2-6.

29. Satsangi J, Silverberg MS, Vermeire S, Colombel JF. The Montreal classification of inflammatory bowel disease: controversies, consensus, and implications. Gut 2006;55:749-753.

30. Lesage S, Zouali H, Cézard JP, et al; GETAID Group. CARD15/ NOD2 mutational analysis and genotype-phenotype correlation in 612 patients with inflammatory bowel disease. Am J Hum Genet 2002;70:845-857.

31. Eckmann L, Karin M. NOD2 and Crohn's disease: loss or gain of function? Immunity 2005;22:661-667.

32. Parente F, Pastore L, Bargiggia S, et al. Incidence and risk factors for gallstones in patients with inflammatory bowel disease: a large case-control study. Hepatology 2007;45:1267-1274.

33. Zhang FM, Xu CF, Shan GD, Chen HT, Xu GQ. Is gallstone disease associated with inflammatory bowel diseases? A meta-analysis. $J$ Dig Dis 2015;16:634-641.

34. Christodoulou DK, Katsanos KH, Kitsanou M, Stergiopoulou C, Hatzis J, Tsianos EV. Frequency of extraintestinal manifestations in patients with inflammatory bowel disease in Northwest Greece and review of the literature. Dig Liv Dis 2002;34:781-786.

35. van Sommeren S, Janse M, Karjalainen J, et al. Extraintestinal manifestations and complications in inflammatory bowel disease: from shared genetics to shared biological pathways. Inflamm Bowel Dis 2014;20:987-994.

36. Gazouli M, Pachoula I, Panayotou I, et al. NOD2/CARD15, ATG16L1 and IL23R gene polymorphisms and childhood-onset of Crohn's disease. World J Gastroenterol 2010;16:1753-1758.

37. Nuij VJAA, Peppelenbosch MP, van der Woude CJ, Fuhler GM. Genetic polymorphism in ATG16L1 gene is associated with adalimumab use in inflammatory bowel disease. J Transl Med;15:248. 